Preprint DM-FCT-UNL

\title{
The idempotent-separating degree of a block-group
}

\author{
Vítor H. Fernandes ${ }^{1}$ \\ Departamento de Matemática, F.C.T., Universidade Nova de Lisboa, 2829-516 \\ Monte da Caparica, Portugal; also: Centro de Álgebra, Universidade de Lisboa, \\ Av. Prof. Gama Pinto 2, 1649-003 Lisboa, Portugal; e-mail: vhf@fct.unl.pt
}

\begin{abstract}
In this paper we describe the least non-negative integer $n$ such that there exists an idempotent-separating homomorphism from a finite block-group $S$ into the monoid of all partial transformations of a set with $n$ elements. In particular, as for a fundamental semigroup $S$ this number coincides with the smallest size of a set for which $S$ can be faithfully represented by partial transformations, we obtain a generalization of Easdown's result established for fundamental finite inverse semigroups.
\end{abstract}

2000 Mathematics subject classification: 20M10, 20M20, 20M30.

\section{Introduction and preliminaries}

It is well-known that any semigroup can always be represented faithfully by full or partial transformations of a set. Given a finite semigroup $S$, it is then natural to ask for a description of the least non-negative integer $n$ such that $S$ can be embedded in $\mathcal{P} \mathcal{T}_{n}$, the monoid (under composition) of all partial transformations of a set with $n$ elements. This question derives from Problem 45 posed by Schein in 1972 [11] and was solved by Easdown in 1987 [2] for fundamental finite inverse semigroups. By a fundamental semigroup we mean a semigroup without non-trivial idempotent-separating congruences. In this paper, we study a related problem: we consider idempotent-separating representations by partial transformations, instead of faithful representations, and describe the least non-negative integer $n$ such that there exists an idempotentseparating homomorphism from a block-group $S$ into $\mathcal{P} \mathcal{T}_{n}$. Since for a fundamental semigroup $S$ this number coincides with the smallest size of a set for which $S$ can be faithfully represented by partial transformations, in particular we generalize the result of Easdown. Recall that a block-group can be defined as a finite semigroup whose elements have at most one inverse. For convenience, we will also call block-group to any infinite semigroup such that

\footnotetext{
${ }^{1}$ The author gratefully acknowledges support of FCT and FEDER, within the project POCTI-ISFL-1-143 of CAUL, and the fellowship SFRH/BSAB/244/2001.
} 
every element has at most one inverse. A block-group can also be defined as a semigroup such that every $\mathcal{R}$-class and every $\mathcal{L}$-class has at most one idempotent. Clearly, the class of all block-groups comprises all idempotent commuting semigroups and, in particular, all inverse semigroups. The class BG of all finite block-groups forms a pseudovariety of semigroups (i.e. a class of finite semigroups closed under homomorphic images of subsemigroups and finitary direct products) and plays a main role in the following celebrated result: $\nabla \mathrm{G}=\mathrm{PG}=\mathrm{J} * \mathrm{G}=\mathrm{J} \otimes \mathrm{G}=\mathrm{BG}=\mathrm{EJ}$, where $\mathrm{G}$ and $\mathrm{J}$ denote the pseudovarieties of all groups and of all $\mathcal{J}$-trivial semigroups, respectively, $P G$ and $\diamond \mathrm{G}$ denote the pseudovarieties generated by all power monoids of groups and by all Schützenberger products of groups, respectively, and, finally, EJ denotes the pseudovariety of all semigroups whose idempotents generate a $\mathcal{J}$ trivial semigroup. See [9] for precise definitions and a complete story of these equalities.

Given a set $X$, we denote by $\mathcal{P} \mathcal{T}(X)$ the monoid (under composition) of all partial transformations on $X$ and by $\mathcal{I}(X)$ the symmetric inverse semigroup on $X$, i.e. the inverse submonoid of $\mathcal{P} \mathcal{T}(X)$ of all injective partial transformations on $X$.

A well-known result due to Vagner and Preston states that every inverse semigroup $S$ has a faithful representation into $\mathcal{I}(S)$. Another classical representation of an inverse semigroup is the Munn representation. We may describe it as follows: for every inverse semigroup $S$, denoting by $E$ the semilattice of all idempotents of $S$, a homomorphism from $S$ into $\mathcal{I}(E)$ is defined by

$$
\begin{aligned}
& \phi: S \rightarrow \mathcal{I}(E) \\
& s \mapsto s \phi: E s s^{-1} \rightarrow E s^{-1} s \\
& \text { e } \mapsto s^{-1} e s .
\end{aligned}
$$

Notice that, in general, the Munn representation of an inverse semigroup is not injective. Indeed, the kernel of $\phi$ is the maximum idempotent-separating congruence on $S$. Therefore, $\phi$ is an injective homomorphism if and only if $S$ is a fundamental semigroup (see [6] or [8], for more details).

For a finite semigroup $S$, a description of the maximum idempotent-separating congruence on $S$ was first presented by Rhodes in [10] (see also Chapter 8 of Arbib's book [1]). Next, we recall this description, using a slightly different language. Let $S$ be an arbitrary semigroup. Let $R L(S)$ and $R R(S)$ be the set of all regular $\mathcal{L}$-classes of $S$ and the set of all regular $\mathcal{R}$-classes of $S$, respectively, and denote the dual semigroup of $\mathcal{P} \mathcal{T}(R R(S))$ by $\mathcal{P} \mathcal{T}^{*}(R R(S))$. Consider the homomorphism $\Theta: S \rightarrow \mathcal{P} \mathcal{T}\left(R L(S) \times \mathcal{P} \mathcal{T}^{*}(R R(S)), s \mapsto\left(\rho_{s}, \lambda_{s}\right)\right.$, where the partial map $\rho_{s}$ is defined by

$$
\begin{aligned}
\rho_{s}: R L(S) & \rightarrow R L(S) \\
L_{x} & \mapsto \begin{cases}L_{x s} & \text { if } x s \mathcal{R} x \\
\text { undefined } & \text { otherwise }\end{cases}
\end{aligned}
$$


and the partial map $\lambda_{s}$ is defined dually, for all $s \in S$. Then, for a finite semigroup $S$, Rhodes proved that the kernel of $\Theta$ is the maximum idempotentseparating congruence on $S$. More generally, this result is also valid for an eventually regular semigroup $S$, i.e. a semigroup $S$ such that each element of $S$ has a power which is regular (see [3]). Notice that Rhodes considered $\mathcal{H}^{\prime}$ homomorphisms (i.e. a homomorphism such that all pairs of regular elements of its kernel are $\mathcal{H}$-related) instead of idempotent-separating homomorphisms: it is easy to show that these two notions coincide for eventually regular semigroups. Let $\mu$ be the kernel of $\Theta$. Clearly, $\mu$ can be described by: given $a, b \in S, a \mu b$ if and only if, for each regular element $x \in S$ the following four conditions are satisfied: $x \mathcal{R} x a$ implies $x a \mathcal{H} x b ; x \mathcal{R} x b$ implies $x a \mathcal{H} x b ; x \mathcal{L} a x$ implies $a x \mathcal{H} b x$; and $x \mathcal{L} b x$ implies $a x \mathcal{H} b x$. Observe that, in general for an arbitrary semigroup $S$, the relation $\mu$ is an idempotent-separating congruence.

We assume some knowledge on semigroups, namely on Green's relations, regular elements and inverse semigroups. Possible references are [6, 8].

\section{Idempotent-separating representations of a block-group}

Let $S$ be a semigroup. We denote by $E(S)$ the set of all idempotents of $S$ and by $\operatorname{Reg}(S)$ the set of all regular elements of $S$. Recall the definition of the quasiorders $\leq_{\mathcal{R}}$ and $\leq_{\mathcal{L}}$ associated to the Green relations $\mathcal{R}$ and $\mathcal{L}$, respectively: for all $s, t \in S, s \leq_{\mathcal{R}} t$ if and only if $s S^{1} \subseteq t S^{1}$ and $s \leq_{\mathcal{L}} t$ if and only if $S^{1} s \subseteq$ $S^{1} t$, where $S^{1}$ denotes the monoid obtained from $S$ through the adjoining of an identity if $S$ has none and denotes $S$ otherwise. To each element $s \in S$, we associate the following two subsets of $E(S): \mathcal{R}(s)=\left\{e \in E(S) \mid e \leq_{\mathcal{R}} s\right\}$ and $\mathcal{L}(s)=\left\{e \in E(S) \mid e \leq_{\mathcal{L}} s\right\}$. Clearly, if $e \in \mathcal{R}(s)$ then $e s \in \operatorname{Reg}(S)$ and, dually, if $e \in \mathcal{L}(s)$ then $s e \in \operatorname{Reg}(S)$.

Now, let $S$ denote a (finite or infinite) block-group and $s^{-1}$ the unique inverse of a regular element $s \in S$. It is easy to check that, given $s \in S$ and $e \in \mathcal{R}(s)$, then $e=(e s)(e s)^{-1}=s(e s)^{-1}=s(e s)^{-1} e,(e s)^{-1}(e s) \in \mathcal{L}(s)$ and $(e s)^{-1}(e s) \mathcal{D} e$. Dually, for $s \in S$ and $e \in \mathcal{L}(s)$, we have also $e=(s e)^{-1}(s e)=$ $(s e)^{-1} s=e(s e)^{-1} s,(s e)(s e)^{-1} \in \mathcal{R}(s)$ and $(s e)(s e)^{-1} \mathcal{D} e$. From these properties, it is easy to deduce that the mappings $\delta_{s}: \mathcal{R}(s) \rightarrow \mathcal{L}(s), e \mapsto(e s)^{-1}(e s)$, and $\bar{\delta}_{s}: \mathcal{L}(s) \rightarrow \mathcal{R}(s), e \mapsto(s e)(s e)^{-1}$, are mutually inverse bijections that preserve $\mathcal{D}$-classes. Moreover, if we identify each regular $\mathcal{L}$-class with its unique idempotent, it is not difficult to prove that, given $s \in S, \delta_{s}$ is a restriction of $\rho_{s}$ and, in fact, they coincide if $S$ is eventually regular. Dually, we have the analogous properties by identifying each regular $\mathcal{R}$-class with its unique 
idempotent. It is routine to show that the mapping

$$
\begin{array}{rllll}
\delta: S & \rightarrow \mathcal{I}(E(S)) & & & \\
s & \mapsto \quad \delta_{s}: & \mathcal{R}(s) & \rightarrow & \mathcal{L}(s) \\
& & & e & \mapsto(e s)^{-1}(e s)
\end{array}
$$

is an idempotent-separating homomorphism. Clearly, for an inverse semigroup $S$, the homomorphism $\delta$ defined above coincides with the (usual) Munn representation of $S$. Therefore, for a block-group $S$, it seems appropriate to also call this homomorphism the Munn representation of $S$. Furthermore, like for the Munn representation of an inverse semigroup, as a particular instance of Rhodes' result (notice that, for an eventually regular block-group $S$, up to an identification, $\rho_{s}$ and $\lambda_{s}$ are mutually inverse bijections, for all $s \in S$ ), we immediately have:

Theorem 1.1 The kernel of the Munn representation of a block-group contains $\mu$. Moreover, if $S$ is an eventually regular block-group then the kernel of the Munn representation of $S$ is the maximum idempotent-separating congruence on $S$.

Notice the following immediate interesting consequence of Theorem 1.1:

Corollary 1.2 Every fundamental block-group is a subsemigroup of an inverse semigroup.

Observe that we defined a semigroup $S$ to be fundamental if the identity is the unique idempotent-separating congruence on $S$. This concept extends naturally the notion of "fundamental" from regular to arbitrary semigroups and was proposed by Edwards in [4]. An alternative definition was considered by Grillet [5]: for this author a semigroup is "fundamental" if the maximum congruence contained in $\mathcal{H}$ is the identity. Clearly, this last notion comprises the first one, since a congruence contained in $\mathcal{H}$ separates idempotents. It is well known that for regular semigroups the converse is also true, i.e. these two notions of "fundamental" coincide. This is not the case in general, even for finite semigroups. For instance, if $S$ is a null semigroup (i.e. a semigroup with zero 0 such that $\left.S^{2}=\{0\}\right)$ then $S$ is $\mathcal{H}$-trivial and all congruences on $S$ separates idempotents (there exists just one: the zero), whence if $S$ has at least two elements, the maximum congruence on $S$ contained in $\mathcal{H}$ is the identity and $S$ has at least two distinct idempotent-separating congruences.

Now, let $E$ be a finite semilattice. Denote by 0 the zero of $E$ and, for $e, f \in E$, denote by $e \vee f$ the supremum of $e$ and $f$, when it exists, with respect to the natural partial order $\leq$ of $E$. Recall that $e \leq f$ if and only 
if $e=e f(=f e)$. Let $e \in E$. Say that $e$ is join irreducible if $e \neq 0$ and, for all $e_{1}, e_{2} \in E, e=e_{1} \vee e_{2}$ implies $e=e_{1}$ or $e=e_{2}$. Given a subset $X$ of $E$, we denote the set of all join irreducible elements of $E$ belonging to $X$ by $\operatorname{Irr}(X)$. Observe that, any element $e \in E$ is the supremum of the set of all join irreducible elements of the principal ideal generated by $e$, i.e. $e=\bigvee \mathfrak{I r r}(\{f \in E \mid f \leq e\})$ (see [2, Lemma 3]).

Example 1.1 Let $n \in \mathbb{N}, \mathcal{I}_{n}=\mathcal{I}(\{1,2, \ldots, n\})$ and $E=E\left(\mathcal{I}_{n}\right)$. Since $E$ is the set of all partial identities on $\{1,2, \ldots, n\}$ and, for all $\alpha, \beta \in E, \alpha \leq \beta$ if and only if $\alpha$ is a restriction of $\beta$, we have $\mathfrak{I r r}(E)=\left\{\left(\begin{array}{l}1 \\ 1\end{array}\right),\left(\begin{array}{l}2 \\ 2\end{array}\right), \ldots,\left(\begin{array}{l}n \\ n\end{array}\right)\right\}$ and so $|\mathfrak{I} \mathfrak{r r}(E)|=n$.

Next, recall that, given any semigroup $S$, the natural partial order $\leq$ of $E(S)$ is defined by $e \leq f$ if and only if $e=e f=f e$, for all $e, f \in E(S)$. Recall also that BG $=\llbracket\left(x^{\omega} y^{\omega}\right)^{\omega}=\left(y^{\omega} x^{\omega}\right)^{\omega} \rrbracket=\llbracket\left(x^{\omega} y^{\omega}\right)^{\omega} x^{\omega}=\left(x^{\omega} y^{\omega}\right)^{\omega}=y^{\omega}\left(x^{\omega} y^{\omega}\right)^{\omega} \rrbracket$ (see [9]). Using these descriptions, given $S \in \mathrm{BG}$, it is easy to show that $(E(S), \leq)$ is a meet-semilattice. In fact, the infimum $e \wedge f$ of $e$ and $f$ is equal to $(e f)^{\omega}$, for all $e, f \in E(S)$.

Let us consider the Munn representation $\delta: S \rightarrow \mathcal{I}(E(S))$ of $S \in \mathrm{BG}$, as defined above. Let $s \in S, e \in E(S)$ and $a, b \in E(S)$ be such that $e=$ $a \vee b$. If $e \in \mathcal{R}(s)$ then, it is easy to show that, $a, b \in \mathcal{R}(s)$ and $e \delta_{s}=$ $a \delta_{s} \vee b \delta_{s}$. Dually, if $e \in \mathcal{L}(s)$ then $a, b \in \mathcal{L}(s)$ and $e \bar{\delta}_{s}=a \bar{\delta}_{s} \vee b \bar{\delta}_{s}$. Now, it is a routine matter to prove that, given $s \in S$, the mappings $\delta_{s}$ and $\bar{\delta}_{s}$ preserve join irreducible idempotents and so we may consider their restrictions $\vartheta_{s}: \mathfrak{I r r}(\mathcal{R}(s)) \rightarrow \mathfrak{I r r}(\mathcal{L}(s)), e \mapsto(e s)^{-1}(e s)$, and $\bar{\vartheta}_{s}: \mathfrak{I r r}(\mathcal{L}(s)) \rightarrow \mathfrak{I r r}(\mathcal{R}(s))$, $e \mapsto(s e)(s e)^{-1}$, respectively, which are mutually inverse bijections.

We can now prove the main result of this section.

Theorem 1.3 Let $S$ be a finite block-group. Then, the mapping $\vartheta: S \rightarrow$ $\mathcal{I}(\mathfrak{I r r}(E(S))), s \mapsto \vartheta_{s}$, is an idempotent-separating homomorphism. Moreover, the kernel of $\vartheta$ is the maximum idempotent-separating congruence on $S$.

Proof. Clearly, $\vartheta$ is a homomorphism. Next, we prove that $\vartheta$ separates idempotents. Let $e, f \in E(S)$ be such that $\vartheta_{e}=\vartheta_{f}$. Hence, in particular, $\mathfrak{I} \mathfrak{r r}(\mathcal{R}(e))=\mathfrak{I} \mathfrak{r r}(\mathcal{R}(f))$. Since $\mathcal{R}(e)$ and $\mathcal{R}(f)$ are the principal ideals of $(E(S), \wedge)$ generated by $e$ and $f$, respectively, it follows that $e=\bigvee \mathfrak{I} \mathfrak{r}(\mathcal{R}(e))=$ $\bigvee \mathfrak{I r} \mathfrak{r}(\mathcal{R}(f))=f$. Thus, $\vartheta$ is an idempotent-separating homomorphism. Finally, as the kernel of $\delta$ is contained in the kernel of $\vartheta$, then we immediately conclude, by Theorem 1.1, that the kernel of $\vartheta$ is the maximum idempotentseparating congruence on $S$, as required. 


\section{The idempotent-separating degree of a block-group}

Let $S$ be a finite semigroup. We define the idempotent-separating degree $\mathfrak{d}(S)$ of $S$ to be the least non-negative integer $n$ such that there exists an idempotentseparating homomorphism from $S$ into $\mathcal{P} \mathcal{T}_{n}$. The minimal faithful degree $\mathrm{d}(S)$ of $S$ is the least non-negative integer $n$ such that $S$ can be embedded in $\mathcal{P} \mathcal{T}_{n}$. For a finite fundamental inverse semigroup $S$, Easdown [2] proved that $\mathrm{d}(S)$ is the number of join irreducible elements of $E(S)$. In this section, we will show that, for any $S \in \mathrm{BG}, \mathfrak{d}(S)$ is also the number of join irreducible elements of $E(S)$.

Notice that, for an arbitrary finite semigroup $S$, since $S / \mu$ is fundamental [4], we have $\mathfrak{d}(S) \leq \mathfrak{d}(S / \mu)=\mathrm{d}(S / \mu)$. It will follow from our result that, for $S \in \mathrm{BG}, \mathfrak{d}(S)=\mathrm{d}(S / \mu)$. This equality is not true in general, as our next example shows.

Example 2.1 Let $L C_{0}$ [7] be the subsemigroup of $\mathcal{P} \mathcal{T}_{2}$ generated by the idempotents $\left(\begin{array}{l}12 \\ 11\end{array}\right),\left(\begin{array}{l}1 \\ 1\end{array}\right)$ and $\left(\begin{array}{l}2 \\ 2\end{array}\right)$. Then $L C_{0}$ is a five elements semigroup with just one non-regular element that forms a 0 -minimal $\mathcal{J}$-class. It is clear that $\mathfrak{d}\left(L C_{0}\right)=2=\mathrm{d}\left(L C_{0}\right)$. On the other hand, $L C_{0} / \mu$ is a four elements band and, by inspection, it is easy to verify that $L C_{0} / \mu$ cannot embed in $\mathcal{P} \mathcal{T}_{2}$, whence $\mathrm{d}\left(L C_{0} / \mu\right) \geq 3$.

For $\alpha \in E\left(\mathcal{P} \mathcal{T}_{n}\right)$, denote by $\bar{\alpha} \in E\left(\mathcal{I}_{n}\right)$ the partial identity with domain $\operatorname{Im}(\alpha)$. For any two idempotents $\alpha$ and $\beta$ of $\mathcal{P} \mathcal{T}_{n}$, it is routine to show that: (i) if $\alpha \leq \beta$ then $\bar{\alpha} \leq \bar{\beta}$; (ii) $\bar{\alpha} \bar{\beta} \leq \overline{(\alpha \beta)^{\omega}}$ and $\bar{\alpha} \leq \overline{(\beta \alpha)^{\omega}}$; and (iii) if $\overline{(\alpha \beta)^{\omega}} \leq \bar{\alpha}, \bar{\beta}$ then $\bar{\alpha} \bar{\beta}=\overline{(\alpha \beta)^{\omega}}$. Now, it is easy to prove that, if $T \in \mathrm{BG}$ is a subsemigroup of $\mathcal{P} \mathcal{T}_{n}$, then the mapping $(E(T), \wedge) \rightarrow\left(E\left(\mathcal{I}_{n}\right), \cdot\right), \alpha \mapsto \bar{\alpha}$, is an injective homomorphism of semilattices.

Next, we prove the main result of this paper.

Theorem 2.1 The idempotent-separating degree of a finite block-group is equal to its number of join irreducible idempotents.

Proof. Let $S$ be a finite block-group. Since the map $\vartheta: S \rightarrow \mathcal{I}(\mathfrak{I r r}(E(S)))$, defined in the Theorem 1.3, is an idempotent-separating homomorphism, we have $\mathfrak{d}(S) \leq|\mathfrak{I} \mathfrak{r r}(E(S))|$. On the other hand, let $\varphi: S \rightarrow \mathcal{P} \mathcal{T}_{\mathfrak{d}(S)}$ be an idempotent-separating homomorphism. Then the mapping $(E(S), \wedge) \rightarrow$ $\left(E\left(\mathcal{I}_{\mathfrak{o}(S)}\right), \cdot\right), e \mapsto \overline{e \varphi}$, is an injective homomorphism of semilattices. As a semilattice is a fundamental inverse semigroup, by Easdown's result, we have $|\mathfrak{I} \mathfrak{r r}(E(S))| \leq \mathfrak{d}(S)$, as required.

Corollary 2.2 If $S$ is a finite block-group then $\mathfrak{d}(S)=\mathrm{d}(S / \mu)$. 


\section{Acknowledgments}

The author wishes to thank M.V. Volkov and the anonymous referees for many useful comments and suggestions which improved substantially this paper.

\section{References}

[1] Arbib, M., "Algebraic Theory of Machines, Languages and Semigroups", Academic Press, New York, 1968.

[2] Easdown, D., The minimal faithful degree of a fundamental inverse semigroup, Bull. Austral. Math. Soc. 35 (1987), 373-378.

[3] Edwards, P.M., Eventually regular semigroups, Bull. Austral. Math. Soc. 28 (1983), 23-38.

[4] Edwards, P.M., Fundamental semigroups, Proc. Royal Soc. Edinburgh 99A (1985), 313-317.

[5] Grillet, P.A., "Semigroups, an Introduction to the Structure Theory", Marcel Dekker, Inc, 1995.

[6] Howie, J.M., "Fundamentals of Semigroup Theory", Oxford University Press, 1995.

[7] Lee, E.W.H., and M.V. Volkov, On the structure of the lattice of combinatorial Rees-Sushkevich varieties, Semigroups and Formal Languages, eds. J. André et al., World Scientific (to appear).

[8] Petrich, M., "Inverse Semigroups", John Wiley \& Sons, 1984.

[9] Pin, J.-E., BG = PG: a success story, Semigroups, Formal Languages and Groups, ed. J. Fountain, Kluwer Academic Pub., (1995), 33-47.

[10] Rhodes, J., Some results on finite semigroups, J. Algebra 4 (1966), 471504.

[11] Shevrin, L.N., The Sverdlovsk Tetrad, Semigroup Forum 4 (1972), 274280. 